\title{
Evaluation of ovarian cysts in adolescents
}

\author{
Jessica R. Zolton, Priya B. Maseelall \\ Department of Obstetrics and Gynecology, Summa Akron City Hospital, Akron, USA \\ Email: zoltonj@summahealth.org
}

Received 26 June 2013; revised 25 July 2013; accepted 1 August 2013

Copyright (c) 2013 Jessica R. Zolton, Priya B. Maseelall. This is an open access article distributed under the Creative Commons Attribution License, which permits unrestricted use, distribution, and reproduction in any medium, provided the original work is properly cited.

\begin{abstract}
The presentation of an adnexal mass in an adolescent is a concerning event for the patient and the family. Patients are most likely to present with abdominal pain or a palpable mass. The diagnostic imaging of choice is pelvic ultrasonography. The majority of these lesions are benign ovarian cysts. These cysts should be followed with ultrasound, as many will spontaneously regress. Further evaluation with laboratory tests may also be warranted, which will aid in diagnosis and treatment. Ovarian cysts that are failed to resolve, severely symptomatic, or concerned with malignancy require surgical intervention. Laparoscopy has shown to be beneficial in the adolescent population and should be the procedure of choice. In addition, ovarian conservation is the ideal treatment in order to continue normal pubertal development and preserve reproductive health.
\end{abstract}

Keywords: Adolescent; Ovarian Cyst; Laparoscopy; Ovarian Torsion; Ultrasonography

\section{INTRODUCTION}

Adnexal masses are uncommon occurrences in the adolescent population. However, when they occur, they are anxiety-provoking for both the patient and her family. Many practitioners are unfamiliar with the proper management of these adnexal masses and are quick to proceed with surgical intervention that is often unnecessary. The estimated incidence of adnexal masses in the adolescent population is approximately 2.6 per 100,000 girls younger than 18 years of age [1]. An estimated ten percent of pediatric ovarian masses are found to be malignant $[2,3]$. Ovarian malignancies account for only one percent of all malignancies found in female patients less than 15 years of age [4]. While the discovery of an ovarian mass in an adolescent patient is concerned, conservative management is warranted due to the fact that the majority of these tumors are benign [5]. It is important to be aware of the presentation, evaluation, and treatment of benign ovarian cysts in order to conserve ovarian function.

\section{ETIOLOGY OF OVARIAN CYSTS}

Ovarian masses are categorized as functional cysts, benign neoplasms, or malignant neoplasms. In a review of females under the age of 21 undergoing surgery for an adnexal mass, $57.9 \%$ of the cases were diagnosed with an ovarian cyst [5]. The prepubertal adolescent is at risk of developing functional cysts due to the failure of involution of follicles [6]. Prepubertal cysts are commonly caused by gonadotropin stimulation of the ovary by the immature hypothalamic-pituitary axis [7]. Millar, et al. found ovarian cysts in $2 \%-5 \%$ of prepubertal females undergoing ultrasound [7]. These cysts are mostly small $(<1 \mathrm{~cm})$ and insignificant. In the postpubertal adolescent, cysts result from failure of ovulation or persistence of ovarian follicles [6].

\section{PRESENTATION}

Ovarian masses present in many ways including abdominal pain, palpable abdominal mass, nausea, vomiting, increasing abdominal girth, and precocious puberty $[2,8]$. A review by Kanizsai, et al. demonstrated that in the adolescent population, cysts are most likely to be associated with irregular menstrual cycles [9]. In prepubertal females, an ovarian cyst most commonly presents as a palpable abdominal mass [2]. Ovarian torsion should be suspected for patients who present with a sudden onset of severe abdominal pain, fever, nausea, and vomiting [10].

Clinical symptoms have not shown to be reliable in the prediction of ovarian malignancy, although increased index of suspicion is warranted for patient who present with an asymptomatic palpable abdominal mass or signs and symptoms of precocious puberty [2,3,5,8]. Age at presentation may also pose a significant risk factor for malignancy, as Oltmann, et al. found a 3-fold greater 
odds ratio for malignancy in females aged 1- to 8-year compared to the 15- to 19-year-old group [2]. This study involved 424 patients with pediatric ovarian masses and found that $22 \%$ of patients aged 1 to 8 years were found to have a malignancy compared to $10 \%$ of patients aged 9 to 15 years [2].

\section{DIFFERENTIAL DIAGNOSES}

A thorough history and physical should be obtained and diseases of the genitourinary and gastrointestinal tracts should be ruled out when patients present with abdominal pain or a palpable mass. It is important to discuss menstrual cycle, sexual history, and contraceptive methods to help elucidate the cause of symptoms. A pregnancy test and a complete blood count should be ordered immediately in patients who present with abdominal pain to identify the presence of pregnancy, leukocytosis, anemia, and hemorrhage. Ovarian torsion should be suspected if fever, severe pain, and leukocytosis are present [11]. Surgical emergencies including ectopic pregnancy, appendicitis, and ovarian torsion should be managed appropriately.

\begin{tabular}{cc}
\hline Differential diagnosis of adnexal mass \\
\hline Ovary & Functional Cyst \\
Neoplasm \\
(benign, borderline or malignant) \\
Torsion \\
Endometrioma \\
Ectopic pregnancy \\
Tubo-ovarian abscess \\
Hydlopian Tube \\
Wolffian duct remnants \\
Uterus \\
Mullerian Anomaly
\end{tabular}

\section{IMAGING}

The diagnostic study of choice for an adnexal mass in the adolescent is ultrasonography. Transvaginal ultrasound is preferred if the patient is mature enough to tolerate exam. If not, a transabdominal image will suffice. A common finding on ultrasound is a functional cyst. Functional cysts form when a follicle fails to involute after ovulation and continues to enlarge [6]. Functional cysts are radiologically described as anechoic thin-walled cysts with distal acoustic enhancement [12]. Corpus luteum cysts are distinguished from "simple" or follicular cysts since they often display internal echoes [6]. If hemorrhage within the cyst occurs, the cyst may appear complex or solid [12]. A hemorrhagic cyst will first appear with a homogenous increase in echogenicity and fluid debris within the cyst [13]. The cyst will steadily decrease as the clot lyses [14]. In addition, evaluation of the fluid in pelvis can further correlate pathology, as blood appears echogenic and simple cystic fluid will appear anechoic
[15]. A teratoma is visualized as a heterogeneous cysticsolid ovarian mass less than $8 \mathrm{~cm}$ on ultrasound [16]. An enlarged ovary and increased volume ratio in comparison to the contralateral ovary is indicative of an ovarian torsion [17]. In children, the presence of Doppler flow does not exclude torsion [14].

While cystic lesions are reassuring for a benign process, heterogeneous masses raise concern for malignancy [2]. Ultrasound findings consistent with malignancy include a solid lesion, increased vascular flow, septations of $3 \mathrm{~mm}$ or greater, and presence of ascites [18]. Size of tumor have not been shown to be a predictor of malignancy, although persistent or enlarging cysts are concerning for a malignant process $[9,19]$.

\section{TUMOR MARKERS}

If masses are complex, solid, or concerning for a malignant process, tumor markers are indicated. Unlike adult females, germ cell tumors are the most prevalent malignant ovarian tumor in adolescents and children [5]. Appropriate tumor markers include CA-125, alpha-fetoprotein, serum beta-hCG, estradiol, inhibin B, and lactate dehydrogenase levels [6]. Tumor markers may aid in diagnosis of a germ cell tumor but they have been reported to be elevated in only $54 \%$ of ovarian malignancies and $6.5 \%$ of benign lesions [2].

\section{MANAGEMENT}

Simple cysts greater than $3 \mathrm{~cm}$ should be managed conservatively with monthly ultrasounds to confirm regression [20]. Ovarian cysts typically resolve spontaneously through 3 menstrual cycles [9]. For postpubertal females, timing of the ultrasound during the follicular phase of the cycle immediately after the menstrual cycle will avoid confusion between a follicle, corpus luteum, and persistent cyst [10]. Large ovarian cysts, defined as greater than $5 \mathrm{~cm}$, require weekly ultrasounds [13]. Warner, et al. successfully managed $90 \%$ of children with large ovarian cysts without surgical intervention [13]. Hemorrhagic functional cysts may be confused with a malignant process due to its solid or complex characteristics but should regress in 2 to 8 weeks [21].

\subsection{Medical Intervention}

Recent literature indicates that the use of oral contraceptives for treatment of functional cysts is not beneficial [22]. For postpubertal females, oral contraceptives may be considered in order to prevent the occurrence of ovarian cysts [10].

\subsection{Surgical Intervention}

Management decisions should be based on physical 
exam, ultrasound findings, and laboratory studies. If the cyst does not resolve or decrease in size after 2 - 3 months, the cyst should be considered pathologic and surgical intervention is warranted [20]. Cysts that cause severe symptoms or are greater than $8 \mathrm{~cm}$ may also be candidates for immediate surgical intervention [10]. Warner, et al. demonstrated a need for surgical intervention for large simple cysts (greater than $5 \mathrm{~cm}$ ) if severe symptoms did not resolve in 12 - 24 hours; associated signs and symptoms such as mass effect were involved; if the cyst failed to decrease in size in $1-2$ weeks, or if the diagnosis was questionable [13]. Concern for acute ovarian torsion warrants immediate surgery in order to salvage ovarian tissue

For benign ovarian cysts that require surgical management, laparoscopy has recently been accepted as the procedure of choice for children and adolescents due to the advantages seen in the adult populations [18,23]. If the treating physician is not skilled in laparoscopy, the patient should be referred to a proficient laparoscopic surgeon with experience in pediatric gynecologic laparoscopy. Laparoscopy offers improved cosmetic appearances and decreases in operative time, blood loss, hospital stay, and postoperative pain $[18,24]$. Laparoscopic surgery also contributes to less adhesion formation than laparotomy, which may result in improved fertility outcomes in the future [25]. In a review of 2126 pediatric patients undergoing treatment of benign ovarian masses, use of laparoscopy increased from $32.1 \%$ in 2000 to $57.9 \%$ in 2010 [26]. It is important for gynecologic surgeons to recognize the benefits of laparoscopy and adopt a minimally invasive technique when appropriate.

The visual inspection of the abdomen during laparoscopy has been shown to have a sensitivity of $100 \%$ for diagnosis of malignant tumors [27]. For lesions concerning for malignancy, initial management during laparoscopy should include pelvic washings, inspection of the contralateral ovary, and an intraoperative frozen section [28]. Laparotomy should be considered if there is concern for upstaging a malignancy if rupture occurs or if the size of the tumor will hinder its ability to be removed by laparoscopic means. [24].

Due to the low frequency of malignant tumors in this population, ovarian sparing surgery is the goal [23]. Traditionally treatment consisted of oophorectomy, which is now considered inappropriately aggressive due to the low incidence of malignant ovarian tumors in the pediatric population. In a review of 2126 pediatric patients undergoing treatment of benign ovarian masses, $40 \%$ of cases underwent oopherectomy [26]. The high percentage of patients undergoing oophorectomy illustrates the slow adoption of ovarian conservation in the adolescent population. The consequences of aggressive therapy are usually not apparent until adulthood. The fertility poten- tial of these patients may be decreased, as women with one ovary have been noted to have higher FSH levels and poorer response to ovarian stimulation during assisted reproductive therapy [29]. In addition, there is an increased frequency of women seeking infertility treatment with a history of a single ovary [30].

Cystectomy, fenestration or decompression of the cyst with removal of the cyst wall should be the procedure of choice for benign ovarian cysts [18]. At our institution, the procedure of choice is cystectomy. The surgeon develops a plane of dissection and removes the cyst wall from the ovary. Hemostasis is obtained by bipolar cautery. The specimen is removed with the Endocatch bag. If the specimen is unable to pass through the $10 \mathrm{~mm}$ port, the cyst is aspirated within the Endocatch bag under direct visualization. Specimens that are too large for the Endocatch bag are aspirated with a needle if there is low risk for malignancy. If spillage occurs, then copious irrigation is performed. Chemical peritonitis has been shown to be extremely rare in adult studies; occurring in just $0.2 \%$ of patients with cyst spillage [31]. The recurrence of ovarian cysts in adult literature has been cited as $4 \%$ with cyst excision versus $84 \%$ with simple aspiration [32]. It should be noted that cytologic evaluation of aspirated fluid of ovarian cysts has proven to be unreliable [33].

\subsection{Ovarian Torsion}

Ovarian torsion requires quick diagnosis and immediate surgical intervention in order to preserve the ovary. Adnexal torsion occurs in $2.7 \%$ of all children who present with acute abdominal pain [34]. Adnexal torsion in children is most commonly caused by a benign ovarian cyst or teratoma. In fact, $97 \%$ of torsions are the result of benign pathology [35]. The size of an ovarian cyst has not been shown to correlate with an increased risk of ovarian torsion $[13,35]$. Ovarian torsions are more likely to result on the right side due to the protective effect of the sigmoid colon [36]. Laparoscopic detorsion of the torsed adnexa is the procedure of choice even if the ovary appears necrotic [37]. Traditionally, surgeons would excise the necrotic appearing ovary but studies have demonstrated that ovarian function does resume in $88 \%$ to $100 \%$ of cases treated conservatively [38]. It is not recommended to perform oophoropexy due to the low risk of subsequent ovarian torsion [39]. In addition, the ovarian cyst or mass should not be removed due to the risk of further damage to the ovary [38]. The patient should be evaluated in 6 - 8 weeks after surgery to determine if secondary surgery is indicated [37].

\section{CONCLUSION}

In conclusion, adolescents and children benefit from 
conservative management of ovarian cysts due to the fact that the majority of cysts are benign. If surgical intervention is necessary, the goal should be to preservation ovarian tissue in order to allow normal pubertal development and preserve fertility. Children and adolescent patients should be referred to a pediatric gynecologist or gynecologic surgeon with knowledge and experience in this topic in order to achieve the best possible outcome.

\section{REFERENCES}

[1] Skinner, M.A., Schlatter, M.G., Heifetz, S.A. and Grosfeld, J.L. (1993) Ovarian neoplasms in children. Archives of Surgery, 128, 849-854.

[2] Oltmann, S.C., Garcia, N., Barber, R., Huang, R., Hicks, B. and Fischer, A. (2010) Can we preoperatively risk stratify ovarian masses for malignancy? Journal of Pediatric Surgery, 45, 130-134.

doi:10.1016/j.jpedsurg.2009.10.022

[3] Cass, D.L., Hawkins, E., Brandt, M.L., Chintaqumpala, M., Bloss, R., Milewicz, A., Minifee, P., Wesson, D., Nuchtern, J. (2001) Surgery for ovarian masses in infants, children, and adolescents: 102 consecutive patients treated in a 15-year period. Journal of Pediatric Surgery, 36, 693-699.doi:10.1053/jpsu.2001.22939

[4] Grovas, A., Fremgen, A., Rauck, A., Ruyman, D., Hutchinson, C., Winchester, D. Menck, H. (1997) The national cancer data base report on patterns of childhood cancers in the United States. Cancer, 80, 2321.

doi:10.1002/(SICI)1097-0142(19971215)80:12<2321::AI D-CNCR14>3.0.CO;2-W

[5] Templeman, C., Fallat, M., Blinchevsky, A. and Hertweck, S. (2000) Noninflammatory ovarian masses in girls and young women. Obstetrics \& Gynecology, 96, 229233.doi:10.1016/S0029-7844(00)00929-7

[6] Laufer, M. and Goldstein, D. (2000) Benign and malignant ovarian masses. Pediatric and adolescent gynecology. Lippincott Williams \& Wilkins, Philadelphia, 685-728.

[7] Millar, D., Blake, J., Stringer, D., Hara, H., Naniak, C. (1993) Prepubertal ovarian cyst formation: 5 years' experience. Obstetrics \& Gynecology, 81, 434-438.

[8] Brown, M., Hebra, A., McGeehin, J., Ross, A. (1992) Ovarian Masses in Children: A review of 91 cases of malignant and benign masses. Journal of Pediatric Surgery, 28, 930-932.doi:10.1016/0022-3468(93)90700-U

[9] Kanizsai, B., Orley, J., Szigetvari, I. and Doszpod, J. (1998) Ovarian cysts in children and adolescents: Their occurrence, behavior, and management. Journal of Pediatric and Adolescent Gynecology, 11, 85-88. doi:10.1016/S1083-3188(98)70117-2

[10] Powell, J.K. (2004) Benign adnexal masses in the adolescent. Adolescent Medicine, 15, 535-547 doi:10.1016/j.admecli.2004.06.008.

[11] Haller, J., Bass, I., Friendman, A. (1984) Pelvic masses in girls: An 8-year retrospective analysis stressing ultrasound as the prime imaging modality. Pediatric Radiology, 14, 363-368.
doi:10.1007/BF02343418

[12] Wu, A. and Siegel, M. (1987) Sonography of pelvic masses in children: Diagnostic predictability. AJR, 148, 1999-2001.doi:10.2214/ajr.148.6.1199

[13] Warner, B.W. (1992) Conservative management of large ovarian cysts in children: The value of serial pelvic ultrasonography. Surgery, 112, 749-755.

[14] Surratt, J. and Siegel, M. (1991) Imaging of pediatric ovarian masses. Radiographics, 11, 533-547.

[15] Hertzberg, B.S., Kliewer, M.A. and Paulson, E.K. (1999) Ovarian cyst rupture causing hemoperitoneum: Imaging features and the potential for misdiagnosis. Abdominal Imaging, 24, 304-308.

[16] Ozcan, E.R., Kuruoglu, S. and Dervisoglu, S. (2013) Ovary-sparing surgery for teratomas in children. Pediatric Surgery International, 29, 233-237. doi:10.1007/s00383-012-3228-X

[17] Servaes, S., Zurakowski, D., Laufer, M., Feins, N., Chow, J. (2007) Sonographic findings of ovarian torsion in children. Pediatric Radiology, 37, 446-451. doi:10.1007/s00247-007-0429-x

[18] Broach, A., Mansuria, S. and Sanfilippo, J. (2009) Pediatric and adolescent gynecologic laparoscopy. Clinical Obstetrics and Gynecology, 52, 380-389. doi:10.1097/GRF.0b013e3181b0bf04

[19] Islam, S., Yamout, S. and Gosche, J. (2008) Management and outcomes of ovarian masses in children and adolescents. Amsurg Surgery, 74, 1062-1065.

[20] Pfeifer, S. and Gosman, G. (1999) Evaluation of adnexal masses in adolescents. Pediatric Clinics of North America, 46, 573-592. doi:10.1016/S0031-3955(05)70138-3

[21] Okai, T., Kobayashi, K., Ryo, E., Kagawa, H., Kozuma, S. and Taketani, Y. (1994) Transvaginal sonographic appearance of hemorrhagic functional ovarian cysts and their spontaneous regression. International Journal of Gynecology \& Obstetrics, 44, 47-52. doi:10.1016/0020-7292(94)90022-1

[22] Grimes, D.A., Jones, L.B., Lopez, L.M., Schulz, S. (2011) Oral contraceptives for functional ovarian cysts. Cochrane Database of Systematic Reviews, 15, CD006134.

[23] Patino, J., Bettolli, M. and Kolberg-Schwerdt, A. (2009) Laparoscopic approach to ovarian mass in children and adolescents: Already a standard in therapy. Journal of Laparoendoscopic \& Advanced Surgical Techniques. Part A, 19, S111-S115.

[24] Michelotti, B., Segura, J., Sau, I., Perez-Bertolez, S. and Prince, J. (2010) Surgical management of ovarian disease in infants, children, and adolescents: A 15-year review. Journal of Laparoendoscopic \& Advanced Surgical Techniques, 15, 261-264. doi:10.1089/lap.2009.0137

[25] Schafer, M., Krahenb, L. and Buchler, M.W. (1998) Comparison of adhesion formation in open and laparoscopic surgery. Digestive Surgery, 15, 148-152. doi:10.1159/000018609

[26] Berger-Chen, S., Herzog, T. and Lewin, S. (2012) Access to conservative surgical therapy for adolescents with benign ovarian masses. Obstetrics \& Gynecology, 119, 270 - 
275. doi:10.1097/AOG.0b013e318242637a

[27] Canis, M. (1994) Laparoscopic diagnosis of adnexal cystic masses: A 12 year experience with long-term follow up. Obstetrics \& Gynecology, 83, 707-712.

[28] Templeman, C. and Fallat, M. (2005) Benign ovarian masses. Seminars in Pediatric Surgery, 14, 93-99. doi:10.1053/j.sempedsurg.2005.01.004

[29] Khalifa, E. (1992) Significance of basal follicle-stimulating hormone levels in women with one ovary in a program of in vitro fertilization. Fertility and Sterility, 57, 835-839.

[30] Lass, A. (1999) The fertility potential of women with a single ovary. Human Reproduction Update, 5, 546-550. doi:10.1093/humupd/5.5.546

[31] Nezhat, C.R., Kalyoneu, S., Nezhat, C.H., Johnson, E., Berlanda, N. and Nezhat, F. (1999) Laparoscopic management of ovarian dermoid cysts: Ten years' experience. JSLS, 3, 179-184

[32] Marana, R. (1996) Operative laparoscopy for ovarian cysts. Excision vs. aspiration. The Journal of Reproductive Medicine, 41, 453-458.

[33] Diernaes, E., Rasmussen, J., Sorensen, T. and Hasch, E. (1987) Ovarian cysts: Management by puncture? Lancet,

\section{1, 1084. doi:10.1016/S0140-6736(87)90503-4}

[34] Hibbard, L. (1985) Adnexal torsion. American Journal of Obstetrics \& Gynecology, 152, 456-461.

[35] Kokoska, E.R., Keller, M.S., Weber, T.R. (2000) Acute ovarian torsion in children. The American Journal of Surgery, 180, 462-465. doi:10.1016/S0002-9610(00)00503-1

[36] Lee, C., Raman, S. and Silvanesaratnam, V. (1989) Torsion of ovarian tumors: A clinicopathological study. International Journal of Gynecology \& Obstetrics, 28, 2125.

[37] Rody, A., Jackish, C. and Klockenbusch, W. (2002) The conservative management of adnexal torsion-A case report and review of the literature. European Journal of Obstetrics \& Gynecology and Reproductive Biology, 101, 83-86.doi:10.1016/S0301-2115(01)00518-8

[38] Oelsner, G. and Shashar D. (2006) Adnexal Torsion. Clinical Obstetrics and Gynecology, 49, 459-463. doi:10.1097/00003081-200609000-00006

[39] Cass, D. (2005) Ovarian torsion. Seminars in Pediatric Surgery, 14, 86-92. doi:10.1053/j.sempedsurg.2005.01.003 